\title{
Perception of relative and absolute age in facial photographs
}

\author{
JOHN B. PITTENGER and ROBERT E. SHAW \\ University of Minnesota, Minneapolis, Minnesota 55455
}

\begin{abstract}
Longitudinal series of photographs of faces of secondary school students were used to evaluate observers' ability to perceive age. Information in individual stimuli was manipulated by masking out parts of the photographs, while information provided by the relations among stimuli was manipulated by task conditions. The ability to perceive relative age was assessed in two tasks requiring subjects to order photographs by age. Absolute age perception was studied in a third task requiring age estimates in years to photographs presented one at a time. While judgments were beyond chance accuracy in all combinations of masking and task conditions, a decrease in either type of information generally produced a decrease in accuracy.
\end{abstract}

Faces as a source of information about a variety of human characteristics have a long tradition in experimental psychology. Darwin's studies of the facial expression of emotion (Darwin, 1859) started a line of research with animals and humans which continues today (cf. Ekman, 1973, for a review). Since Galton's (1879) classic studies of criminality using composite photographs of faces, the face as a source of information for such traits as intelligence (Pintner, 1918 ) and even vocational aptitude (Land is \& Phelps, 1928) have been studied. Facial beauty has been studied by psychologists (Cross, Cross, \& Daly, 1971; lliffe, 1960) and orthodontists (Peck \& Peck, 1970).

Perception of age in faces has, however, been little studied. Study of the area is justified both by the importance of age perception itself and because it bears on other aspects of face perception. First, the perception of age is an important social skill; knowledge of the age of others is a basis for the choice of appropriate social behavior both in human and animal societies. In contrast to the perception of criminality or intelligence, there is a potential source of information for age perception. Since growth produces relatively regular changes in the face, there are correlations between age and facial structure.

Preparation of this paper was supported in part by a Career Development Award to Robert E. Shaw from the National Institute of Child Health and Human Development (1 K04-HD 24010) and by grants to the University of Minnesota, Center for Research in Human Learning, from the National Science Foundation (GB-35703X), the National Institute of Child Health and Human Development (HD-01136 and HD-00098), and the Graduate School of the University of Minnesota. The authors wish to thank Robert J. Arrthun, Michael Weiszel, and the students of the Breck School, Minneapolis, Minnesota, for supplying the photographs used in this study. Requests for reprints should be sent to Robert E. Shaw, Center for Research in Human Learning, 205 Elliott Hall, University of Minnesota, Minneapolis, Minnesota 55455. J. B. Pittenger is presently in the Department of Psychology, University of Arkansas, Little Rock, Arkansas 72204.
Second, many aspects of face perception, such as memory for identity and perception of emotion cannot be understood independent of age. The transformations of growth, which may provide information for age, alter the structures which must be the basis for perception of identity and in which emotion is expressed. How theories of such perceptions should deal with these changes, whether by cognitive "correcting" for age level or by use of some type of invariant structure over growth, remains an open question.

The relevant psychological research on age perception can be reviewed quickly. Markey (1934) reports, as an aside in a study of perceived intelligence in faces, little correlation between estimated and actual age. However, the small stimulus set and lack of procedural details make this study difficult to interpret. Two other studies have shown that profile shape is a sufficient stimulus for age perception. Gardner and Wallach (1965) demonstrated that appropriately exaggerated profiles are seen as extremely old and as extremely young. The present authors have applied spatial coordinate transformations (cf. Thompson, 1942) approximating those produced by growth to profiles and shown that these systematically influence age perception (Pittenger \& Shaw, 1974). Since both of these studies used stimuli that omitted detail of the facial profile such as hairline, skin tone, eyes, nostrils, etc., at least a prima facie case can be made that such features are not necessary to age perception. Similarly, since all facial profiles were normalized for size, the necessity of this information for age perception is likewise ruled out. Mooney (1957), in a study of closure in children, used high contrast, and thus rather distorted and impoverished, photographs of faces and asked children to classify them by age and sex. Although not specifically analyzed in terms of age perception, the results suggest that children can discriminate faces of 
adults from those of children even under considerable stimulus degradation.

The present study assessed age perception from faces using sets of longitudinal photographs of children 11 to 18 years old, a period of rapid growth. Photographs rather than line drawings or sketches were chosen as the most natural practical stimuli. Both relative and absolute age perception and the effects of availability of age information within and anong stimuli were studied. Establishment of such basic results should logically, it seems to us, to precede the study of the effects of particular features of the face on age perception.

We assume that the transformations of the facial structure produced by growth are the major source of stimulus information for the perception of age. Since growth is a process which remodels the shape of the face as a total structure (Enlow, 1968), perceptual information specifying age level should exist in all areas of the face. We attempted to manipulate this information by masking the photographs at three levels: no masking, leaving the entire head visible; masking hair and shoulders, leaving facial outline shape and internal features such as nose shape, mouth shape, and the relations between them visible; and masking hair, shoulders, and outline shape, leaving only internal features visible. If age information is available in a variety of features throughout the face, then for both absolute and relative judgments, increased masking should lessen, but not completely destroy, the accuracy of age perception. This hypothesis was tested in both relative-age tasks, requiring subjects to order photographs by age, and absolute-age tasks, requiring subjects to estimate the age of faces in years.

Growth-produced age information should exist not only in individual faces, but also in the relations among faces. In a set of faces differing in age, two types of relations are possible. If the faces are those of a single individual. the effects of growth have been applied to a single underlying structure and a longitudinal relationship exists. In a set of different individuals, the growth transformations have been applied to different structures and a cross-sectional relationship exists. Longitudinal relations should provide more information for age, and thus afford more accurate perception, than cross-sectional relations. In the former, the basic structure remains invariant over age changes, while in the latter, differences in faces are produced both by age changes and differences in structure. This hypothesis was tested in relative-age tasks by comparing the accuracy of age ordering of faces of a single individual at different ages with those of a number of different individuals at different ages.

The cross-sectional tasks correspond to the age-judgment tasks normally occurring in real life, where different people of various ages are encountered. While longitudinal tasks rarely occur in life, it is obvious that relative age is perceived in albums of family photographs taken over a long period of time and in illustrated biographies. Our hypothesis about growth-produced information predicts that these latter cases should be more accurately perceived than the more common cross-sectional problems.

\section{METHOD}

\section{Design}

Subjects. Ninety University of Minnesota undergraduates. ranging in age from about 18 to 22 years, served as subjects and received course credit for their participation. Ten subjects were assigned randomly to each of the nine task conditions.

Stimulus materials. The stimuli were 90 black-and-white photographs of the head and shoulders of white male students copied from the yearbooks of a private school. For each of 15 individuals, six photographs were copied, one for each grade, 7 through 12 . The age covered in these sets ranged between 12.1 and 19.1 years.

The original photographs were fairly fine-grain, giving good detail of the faces. During the copying procedure, an attempt was made to equalize the overall size of the heads. All views were full face or nearly full face. None had glasses. beards, or moustaches. The photographs were cropped $5.1 \mathrm{~cm}$ wide $x 7.6 \mathrm{~cm}$ high and mounted on white cardboard $7.6 \mathrm{~cm}$ wide $\times 12.7 \mathrm{~cm}$ high.

Task conditions. In the two types of rank ordering tasks, the subjects were presented with six photographs at a time and asked to place the cards in order by age. In the longitudinal tasks. the cards in each set were the six photographs of a single individual, while in the cross-sectional tasks, each set used photographs of six different individuals. Since the photographs were taken on about the same date every year, the ages of the successive faces in each of the longitudinal sets ditfered by almost exactly 1 year. Since the students were born on different dates, age progression with exactly 1-year intervals could not be achieved in the cross-sectional sets. The photographs were grouped into the cross-sectional sets so as to give the closest possible tit to 12 -month intervals. The range of age difference between successive faces was 8 to 21 months, with a mean interval of 12.6 months. Eighty percent of the intervals were between 10 and 14 months.

In the absolute-age task, each subjects was shown the full set of 90 photographs, one photograph at a time, and asked to estimate the person's age. The photographs were shown in different random orders to each subject.

Masking conditions. The amount of information available in each individual photograph was varied by masking out parts of the photograph. Three levels of information were used: the original unniasked photographs showing the full face plus hair and shoulders, and two levels of masking. In the first mask condition, the shoulders and lower neck were painted out, as was all the hair that could be covered without masking the jaw line. This condition was motivated by the fact that there was an obvious correlation between hair length and age. In the second mask condition, all parts of the photograph except the eyes, eyebrows, nose, and mouth were deleted. Performance in the unmasked condition will show accuracy of age perception when maximum information is available. while performance in the second mask condition will show accuracy of perception based only on the internal structure of the face. Comparison of the unmasked with the first mask condition will show the use of information derived from hair length and comparison of the first and second mask conditions will show the use of facial outline information.

All nine combinations of the three task and three masking conditions were tested with 10 different subjects in each combination. Figure 1 shows four sets of photographs, illustrating the various stimulus conditions. 


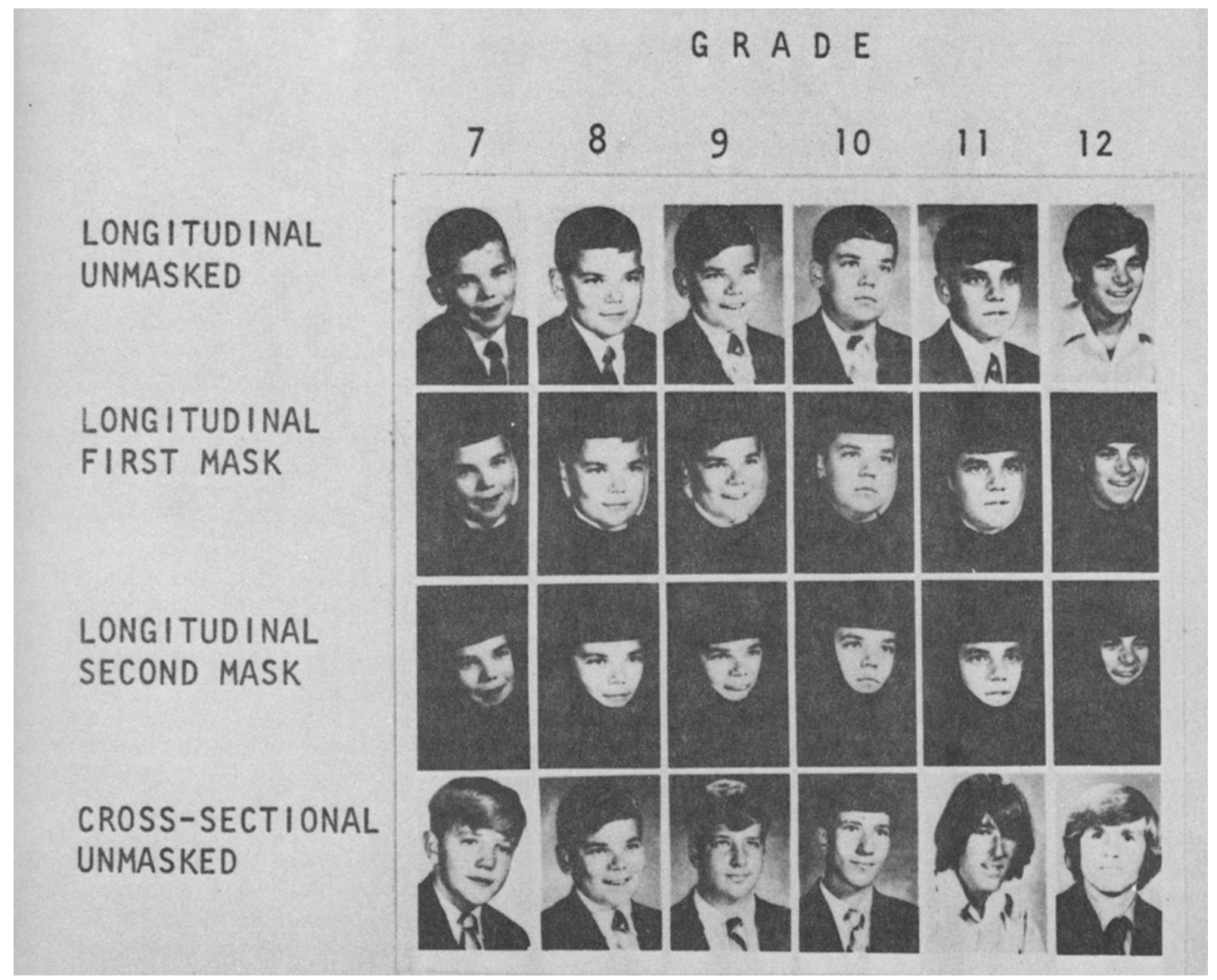

Figure 1. Sample sets of photographs.

\section{Procedure}

For the rank-order conditions, the subjects were instructed that they would be shown sets of photographs of faces and that their task was to order the faces from youngest to oldest. putting the youngest on the left. For the absolute-age condition, they were instructed that they would be shown a series of faces one at a time and that their task was to estimate the ages of the people represented as accurately as possible in years and fractions of years. In nos condition was feedback on accuracy of response provided during the experiment. Photographs were presented on a rack placed on a table in front of the subjects. The experimenter was seated behind the table facing the subject. In all conditions, the subjects were tested individually.

Within each rank order condition, each subject was presented with a different random order of the 15 sets of photographs. Each set of six photographs was shuffled just prior to presentation to the subjects. In the absolute age condition, the set of 90 photographs was thoroughly shuffled for each subject. The orders were checked to avoid two photographs of the same student appearing in succession.

At the end of each testing session, the subjects were asked to describe what characteristics of the faces seemed to them to have influenced their responses.

\section{RESULTS}

A variety of nonparametric statistics were used in the following analyses. For purposes of brevity, the procedures and notation follow those of Siegel (1956), to which the reader is referred for details.

\section{Rank Order Conditions}

Accuracy of ordering. The subjects' ability to perceive relative age was assessed by comparing the observed orderings of the sets of six photographs with the actual-age orders. For each of the six actual-age ranks, mean observed ranks were computed over subjects and photo sets. Figures 2 and 3 portray the relationship between actual and mean observed rank for the six order conditions.

The Kendall tau coefficient was used as the basis for tests of significance. This statistic involves a count of the number of inversions of adjacent items needed 


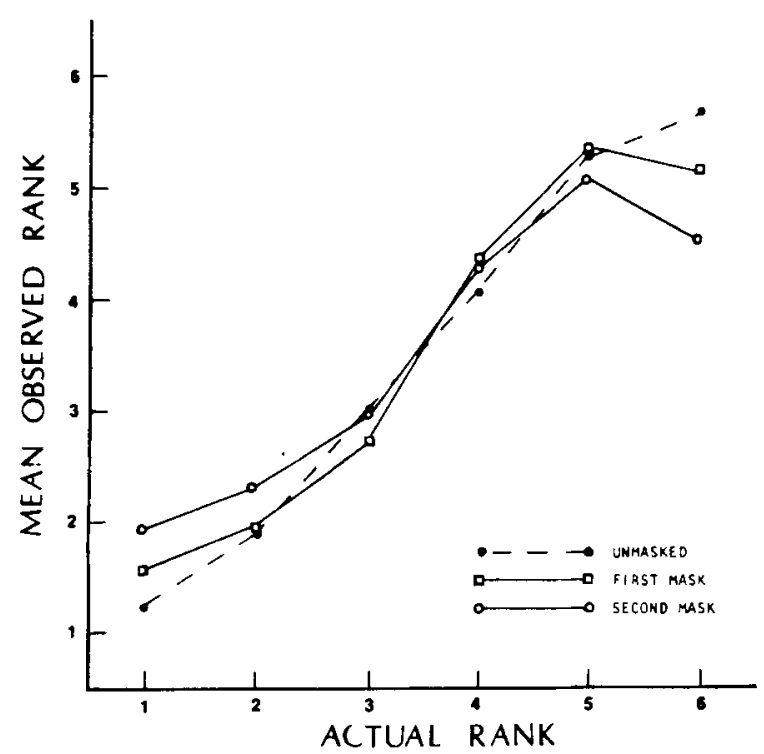

Figure 2. Mean observed ranks, longitudinal rank conditions.

to transform an observed ranking into the correct ranking. Tau coefficients were computed for each subject's ordering of each set of photographs. For each subject. the mean tau over sets was used as a measure of that subject's ability to perceive relative age. For each of the six ordering conditions, the cumulative distribution of the subject's mean tau values was compared with the distribution expected for subjects ordering the photographs randomly with respect to age. Table 1 shows the cumulative distribution expected by chance and those actually observed. It also presents the maximum deviation (D) for the Kolmogorov-Smirnov test. The tests show that the ordering in each condition was better than chance beyond the .01 level of significance.

Differences among order conditions. On the grounds of differences in amount of information available, it was predicted that at each level of

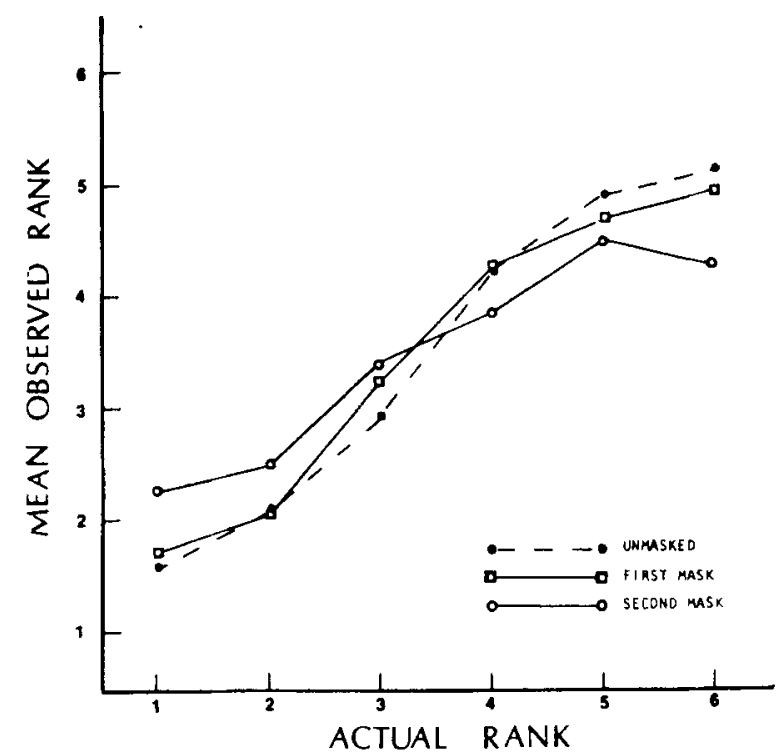

Figure 3. Mean observed ranks, cross-sectional rank conditions.

masking the longitudinal condition would be more accurate than the cross-sectional, and that increased masking would lead to decreased accuracy. These predictions were tested by comparing the distributions of mean tau coefficients in Mann-Whitney $U$ tests. ${ }^{1}$ Table 2 presents the results of these tests. All comparisons except cross-sectional vs. longitudinal at the second level of masking are significantly different in the predicted directions.

Difficulty as a function, of age. Since we claim that growth-produced changes constitute the information for age, we must then predict that ordering according to perceived age level will be most accurate where the process of physical change is most rapid. This possibility was analyzed by finding the distribution of errors in the ordering of adjacent pairs of photographs. Suppose that the correct order of six faces is $1,2,3,4,5.6$, with 1 being the youngest. For

Table 1

Distributions of Cumulative Proportions of Subject's Mean Tau Coefficients

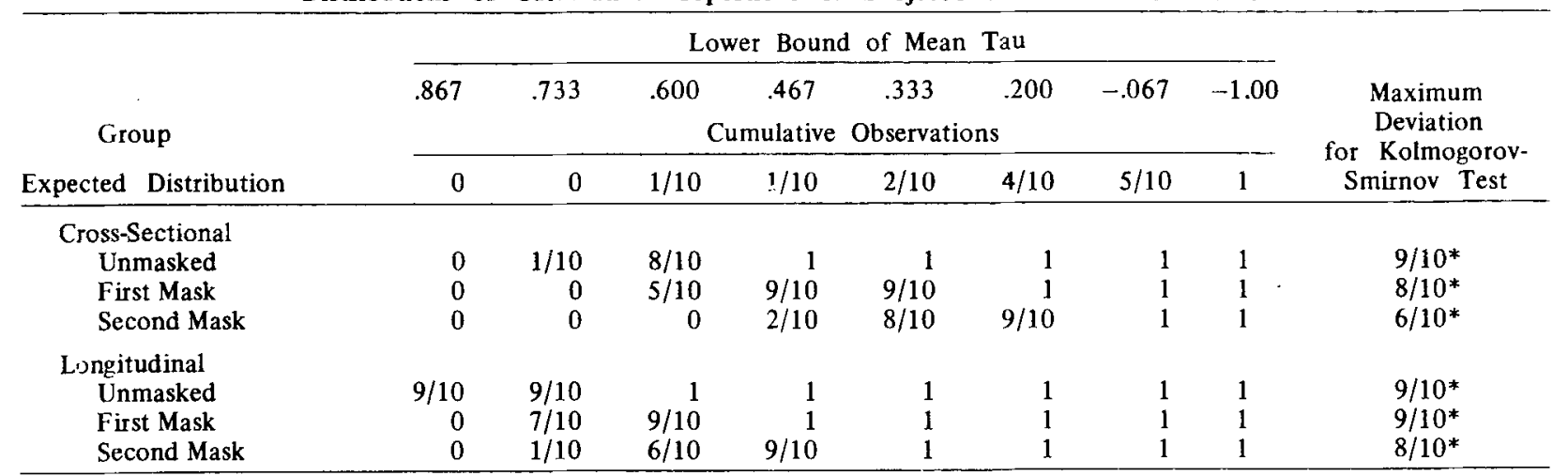

Note-Cell entries are the proportion of subject's mean taus greater than the tau value marked at the top of the column. ${ }_{* p}<.01$ 
Table 2

Comparisons Among Order Conditions

\begin{tabular}{|c|c|c|}
\hline Conditions Compared & $\begin{array}{l}\text { Mar } \\
\text { Whit } \\
\text { UStat }\end{array}$ & $\begin{array}{l}\text { nn- } \\
\text { tney } \\
\text { tistics }\end{array}$ \\
\hline \multicolumn{3}{|l|}{ Within Cross-Sectional } \\
\hline Unmasked vs. First Mask & 25 & * \\
\hline First Mask vs. Second Mask & 9 & $* * *$ \\
\hline \multicolumn{3}{|l|}{ Within Longitudinal } \\
\hline Unmasked vs. First Mask & 8 & $* * *$ \\
\hline First Mask vs. Second Mask & 13.2 & $2 * *$ \\
\hline \multicolumn{3}{|c|}{ Between Cross-Sectional and Longitudinal } \\
\hline Unmasked & 1 & $* * *$ \\
\hline First Mask & 15 & ** \\
\hline Second Mask & 31.5 & \\
\hline
\end{tabular}

Note-For each test $N_{1}=N_{2}=10$. All $p$ values are for one-tailed tests.

$*_{p}<.05 \quad * *_{p}<.01 \quad * * *_{p}<.001$

Table 3

Distributions of Inversions of Adjacent Photographs

\begin{tabular}{|c|c|c|c|c|c|c|}
\hline \multirow[b]{2}{*}{ Condition } & \multicolumn{5}{|c|}{$\begin{array}{c}\text { Mean Inversions Per Subject } \\
\text { Grades }\end{array}$} & \multirow{2}{*}{$\begin{array}{c}x^{2} \text { I Statis- } \\
\text { tic for } \\
\text { Freidman } \\
\text { ANOVA }\end{array}$} \\
\hline & $7-8$ & $8-9$ & $9-10$ & $10-11$ & $11-12$ & \\
\hline \multicolumn{7}{|l|}{ Cross-Sectional } \\
\hline Unmasked & 3.8 & 2.1 & 1.5 & 1.2 & 2.6 & $16.40^{* *}$ \\
\hline First Mask & 2.4 & 1.8 & 1.1 & 1.0 & 1.5 & 7.12 \\
\hline Second Mask & 1.7 & 1.5 & .3 & 1.0 & 1.4 & $11.66^{* *}$ \\
\hline \multicolumn{7}{|l|}{ Longitudinal } \\
\hline Unmasked & 3.3 & 1.1 & .9 & .7 & 4.2 & $16.18 * *$ \\
\hline First Mask & 3.1 & 1.1 & .3 & 1.0 & 5.6 & $24.70^{* * *}$ \\
\hline Second Mask & 2.7 & .9 & 1.0 & 1.1 & 2.6 & $16.66 * *$ \\
\hline
\end{tabular}

Note-Under the hypothesis of no difference among pairs, $\mathrm{X}^{2} r$ is distributed as $\mathrm{X}^{2}(4)$.

${ }_{* p}<.05 \quad * * p<.01$

$* * * p<.001$

the present analysis, we counted only those errors where adjacent photos were reversed in order. Thus, the order $2,1,3,4,5,6$ contains an inversion of 1 and 2 , while $1,2,4,3,6,5$ has inversions of 3 and 4 and of 5 and 6 . The observed order $1,2,5,4,3,6$ would not be scored since the misordering is more complex than inversions of adjacent photographs. Table 3 shows the distribution of errors as a function of grade level. For each condition, the differences in number of errors by location of adjacent pairs is tested by a Friedman analysis of variance by rank on the Errors per Location by Subject.

These results show that difficulty of ordering is not uniform along the age continuum but tends to be easier among the intermediate ages in this group of faces. This is the age range in which puberty occurs and therefore where growth is most rapid.

\section{Absolute-Age Conditions}

Analysis of the absolute-age estimates was based on mean estimates for each subject computed over photographs in blocks of 1 year of actual age. Figure 4 shows the means of these estimates over subjects.
The blocked mean estimates were submitted to an analysis of variance of Age Level by Level of Masking with repeated measures over age levels. This analysis showed significant effects of age level, $F(6,162)=$ 117.94, $\mathrm{p}<.001$, and of level of masking, $F(2,27)=$ 131.97, $\mathrm{p}<.001$, but insignificant Age by Masking interaction, $F(12,162)=1.12, p>.05$. The variance of the estimates was not correlated with actual age: $F_{\max }(3,9)=2.59, \mathrm{p}>.05$. The effect of masking appears to be a tendency for increasing overestimates of age with increased masking. The magnitude of these errors can be estimated by computing the root mean square difference between actual and estimated age. For the unmasked condition, the root mean square difference was .69 years, for the first mask, 1.16 years, and for the second mask, 2.56 years.

Since growth rate is not constant in the period covered by the photographs, but first accelerates then decelerates, we might expect the estimates to be an $S$-shaped rather than linear function of actual age. This possibility was tested by a trend analysis on age with estimates collapsed over levels of masking. There were significant linear, quadratic, and cubic trends; linear, $F(1,162)=686.65, p<.01$; quadratic, $F(1,162)=22.85, p<.01$; cubic, $F(1,162)=26.54$, $\mathrm{p}<.01$; quartic, $\mathrm{F}(1,162)=0.48, \mathrm{p}>.05$.

\section{Subjects' Descriptions of Their Methods}

When asked how they chose their responses, the subjects replied with lists of features which they felt

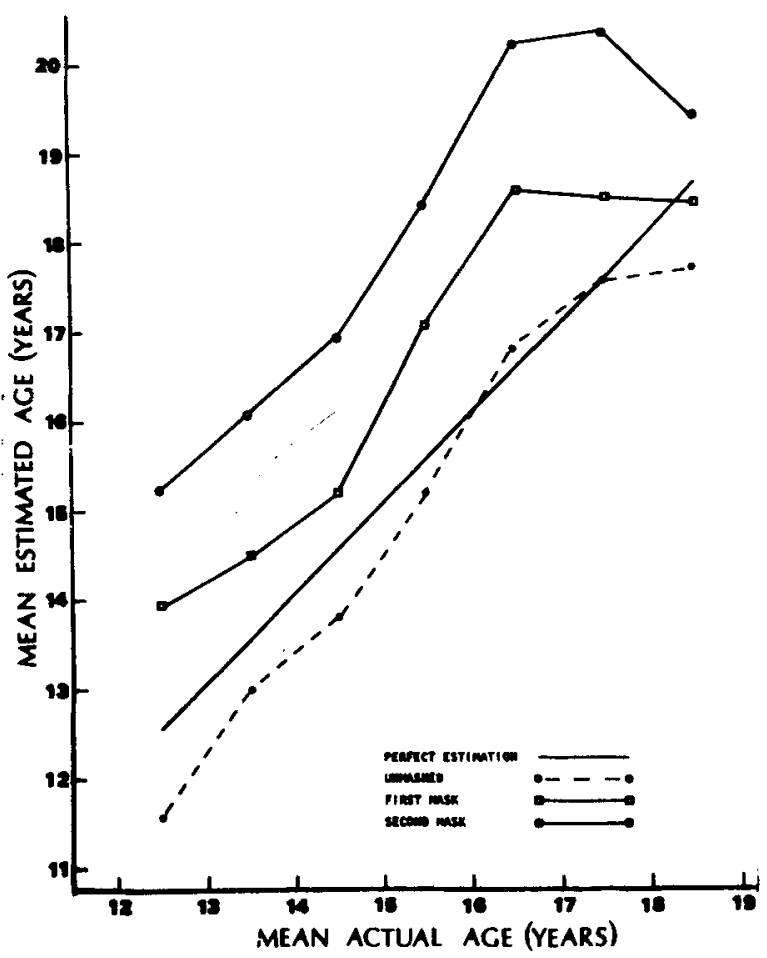

Figure 4. Mean age estimates of the absolute age conditions. Stimull were blocked in 1-year intervals. 
distinguished relative age. A wide variety of teatures was used which included hair length and style. chubbyness of face, presence or absence of freckles, shape of jaw, length of nose. "definition," sharpness, or "development of the face." presence or absence of facial hair, and expression. The last feature is rather interesting in that some subjects claimed that some of the younger faces had an immature, unsophisticated look. While the older ones seemed more mature, "cool." or even cynical. The number of features given by individual subjects varied from as few as one to as many as eight. and there was a great diversity in the sets of features used. While the subjects usually were able to cite features readily, they also admitted that these features were often realized after questioning had started and that they felt there were influences that they could not clearly describe. Subjects in the absolute age condition usually cited features indicating relative rather than absolute age and often claimed that they produced their estimates by comparing the faces to the faces of people whose ages they knew.

\section{DISCUSSION}

The purpose of the present study was to assess the accuracy of relative and absolute age perception using natural stimuli rather than more artificial representations (e.g., outline drawings as in Garnder \& Wallach, 1965, and Pittenger \& Shaw, 1974), and to test hypotheses about the effects of availability of global information within single faces and between groups of faces, rather than study the effects of individual facial features. Within the age range of the stimulus faces, 12 to 19 years, and of the observers, 18 to 22 years, the results show that both absolute and relative age can be perceived with considerable accuracy.

Comparison of the various conditions show that observers are sensitive to variations in available information both in single faces and in the relationships among groups of faces. Within single faces. the effects of masking show that the structural information in the internal configuration of facial features and outline shape plus the social conventions of hair length and style all contribute to age perception. The effect of outline shape confirms with natural stimuli the conclusions of other research based on mathematically transformed outline drawings (Pittenger \& Shaw, 1974). The accuracy of ordering in the cross-sectional rank conditions and of estimates in the absolute conditions shows that observers have some abstract knowledge of the effects of growth, perhaps in the form of a "typical shape" for a given age. However, since stimuli in the cross-sectional conditions were, at least in two of three tests. less accurately ranked than in the longitudinal conditions. it is clear that the availability of a constant underlying structure allows more accurate perception of the growth-produced changes.

The results hold a number of implications for a process theory of age perception. First, it is clear that growth-produced transformations are important. The nature of observer's knowledge of these transformations and their use in perception needs to be specified. In particular, we need to study how information from various parts of the face is integrated, be it in the information pick-up or other stages. Further, we need to know how the invariant structure in longitudinal relations improves detection and use of growthproduced information. Second, the results suggest that the role of a number of factors other than structural changes must be studied. Subjects were apparently using hair length in their estimates. This is a use of knowledge of social conventions in the process. If subjects self-reports are to be trusted, perceived affective characteristics are important. For example, childish vs. mature smiles and naive vs. more cynical attitudes were perceived and reported to be included in the judgments. ${ }^{2}$ Finally, the subjects reported that they also used the conscious cognitive strategy of comparing the faces to be judged with their memories of faces of known age.

\section{REFERENCES}

Cross, J. F.. Cross, J., \& Daly, J. Sex, race, age, and beauty as factors in recognition of faces. Perception \& Psychophysics. $1971,10.393-396$.

DARWIN, C. The expression of the emotions in man and animals. New York: Appleton, 1859. (Reprinted-Chicago: University of Chicago Press, 1965.)

EKMAN, P. (Ed.) Damin and facial expression: $A$ century of research and review'. New York: Academic Press, 1973.

ENLow, D. H. The human face, an account of the postnatal growth and development of the craniofacial skeleton. New York: Hoeber Medical Division, Harper \& Row, 1968.

Galton. F. Generic images. Proceedings of the Royal Institution. $1879,9.161-170$.

Gardener, B. T., \& Wallach, L. Shapes of figures identitied as a baby's head. Perceptual and Motor Skills, 1965, 20. 135-142.

IlifFE. A. H. A study of preferences in feminine beauty. British Journal of Psychology, 1960, 51, 267-273.

Landis, C.. \& Phelps, L. W. The prediction from photographs of success and vocational aptitute. Journal of Experimental Psychology. 1928, 11, 313-324.

MARKEY, F. V. Variations in judgment. Journal of Applied Psychology. 1934. 18. 297-303.

MoOney, C. M. Age in the development of closure ability in children. Canadian Journal of Psychology, 1957, 11, 219-227.

Peck, H., \& PECK, S. A concept of tacial esthetics. Angle Orthodontist, 1970, 40, 284-317.

Pintner, R. Intelligence as estimated from photographs. Psychologicul Revien. 1918, 25. 286-296.

Pittenger, J. B. \& Shaw, R. E. Perceiving age in facial profiles. In J. M. Scandura. J. H. Durin, \& W. H. Wulfeck II (Eds.). 1974 Proceedings: Fifth annual interdisciplinary conference on structural learning. Narbeth. Pa: MERGE Research Institute, 1974.

SIEGEL, S. Nonparametric statistics for the behavioral sciences. New York: McGraw-Hill, 1956 
Thompson, D. A. W. On growth and form (2nd ed.). Cambridge: Cambridge University Press, 1942.

Yin, R. K. Looking at upside-down faces. Journal of Experimental Psychology, 1969, 81, 141-145.

\section{NOTES}

1. In the tests of effect of masking conditions in which longitudinal conditions were compared with longitudinal conditions and cross-sectional with cross-sectional conditions, the subjects mean taus were based on all 15 sets of photographs. When comparing longitudinal with cross-section conditions, however, the taus for the cross-sectional conditions were based on only eight of the photosets. These eight sets were those which had no pair of adjacent photographs differing by more than 12 months. We predicted that the cross-sectional sets would be more difficult, since information for age must be perceived in the relationship among different faces within sets. However, task difficulty should also depend upon the size of the age intervals in the sets. The expected relative difficulty of cross-sectional sets contained intervals of less than 12 months. By using only cross-sectional sets with no interval less than 12 months in the comparison of cross-sectional with longitudinal sets, the prediction of relative difficulty based on information in relations among faces is tested in a conservative fashion.

2. The use of social and affective factors may be pervasive in face perception. In Yin's (1969) study of recognition memory for faces, some subjects seemed to use these factors to form an impression of the faces. It is interesting to note that such a strategy is used even though it can hurt performance. In Yin's study, faces were generally recognized better than other objects. However, when the recognition stimuli were presented upside-down, faces were less accurately recognized than other objects. In the present study, a number of the older faces with broad smiles were frequently rated lower in age than older but more serious faces.

(Received for publication December 2, 1974; revision received May 1, 1975.) 